\title{
Competencias de los estudiantes de Ingeniería en Telecomunicaciones ${ }^{*}$
}

\section{Skills of Telecommunications Engineering students}

Luis Ezequiel Palau De La Rosa**

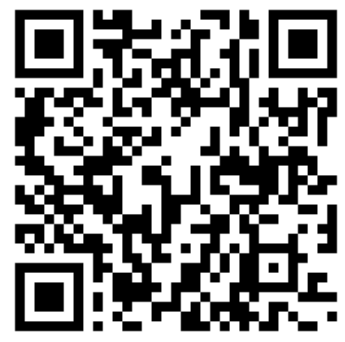

\section{Resumen}

Las empresas de telecomunicaciones demandan profesionales que sean técnicos integrales con dominio de conocimientos en los servicios que se ofertan a sus clientes, con destrezas y habilidades para realizar actividades de instalación y mantenimiento de distintos tipos de servicios (voz, datos y videos). Por ello es importante diagnosticar el nivel de competencias que adquieren los egresados de esta especialidad. El principal propósito de investigación fue obtener información sobre las competencias que logran adquirir los estudiantes de Ingeniería en Telecomunicaciones de la Universidad Católica de Santiago de Guayaquil en contraste con el perfil que demanda la empresa pública y privada de esta área. Se realizó una investigación que empleó metodología cuantitativa. La estrategia de recogida de información se basó en cuestionarios aplicados a estudiantes, profesores y gerentes. Como principales hallazgos se señala que los estudiantes se sienten mejor preparados para: cableado estructurado, desarrollo de proyectos: en diseño y fiscalización. Se concluye que los contenidos de las materias de especialización no abordan temas como: implementación de servicios de banda ancha, instalación y mantenimiento de equipos multiplexores, diseño e implementación de redes ópticas pasivas.

\footnotetext{
* Artículo original derivado del Proyecto "Competencias estudiantiles" Financiado por la Universidad Catolica Santiago de Guayaquil entre junio 2018 y mayo 2019

** Magíster en Sistemas Computacionales. Universidad Católica Santiago de Guayaquil. Guayaquil, Ecuador. E-mail: lepalau@yahoo.com. ORCID: http://orcid.org/0000-0002-5416-0486
}

\author{
Sinergias educativas \\ Enero - junio Vol. 5 -1 - 2020 \\ http://sinergiaseducativas.mx/index.php/revista/ \\ eISSN: 2662-6661 \\ revistasinergia@soyuo.mx \\ Recepción: 04 Septiembre 2018 \\ Aprobación: 16 Abril 2019 \\ Pag 29-45

\section{Disponible en} \\ http://www.redalyc.org/articulo.oa?id=57356143200 \\ 3 \\ Atribución/Reconocimiento-NoComercial- \\ Compartirlgual 4.0 Licencia Pública Internacional - CC


Palabras clave: competencias, perfil profesional, telecomunicaciones, pasantías, curriculum

\begin{abstract}
Telecommunications companies demand professionals who are integral technicians with mastery of knowledge in the services offered to their clients, with skills and abilities to perform installation and maintenance activities of different types of services (voice, data and videos). Therefore it is important to diagnose the level of skills acquired by graduates of this specialty. The main research purpose was to obtain information on the skills that the Telecommunications Engineering students of the Catholic University of Santiago de Guayaquil acquire in contrast to the profile demanded by the public and private companies in this area. An investigation was carried out that used quantitative methodology. The information collection strategy was based on questionnaires applied to students, teachers and managers. The main findings indicate that students feel better prepared for: structured cabling, project development: in design and supervision. It is concluded that the contents of the subjects of specialization do not address issues such as: implementation of broadband services, installation and maintenance of multiplexer equipment, design and implementation of passive optical networks.
\end{abstract}

Key words: competencies, professional profile, telecommunications, internships, curriculum

\title{
Introducción
}

Los avances tecnológicos en el sector de telecomunicaciones, sumado a las nuevas políticas laborales que tienen por objetivo optimizar el recurso humano a ser contratado, ponen en desventaja a los nuevos profesionales al momento de presentarse a las entrevistas de trabajo, por la falta de cumplimiento de los nuevos perfiles profesionales. Las empresas de telecomunicaciones en la actualidad, demandan de las universidades profesionales técnicos integrales, con relación a los conocimientos, destrezas y habilidades para realizar actividades de instalación y mantenimiento de distintos tipos de servicios (voz, datos y videos). Por lo tanto, los centros de formación requieren replantear, el proceso de formación de estos profesionales 
ya que no cumple a cabalidad con los perfiles que solicitan las empresas del sector de telecomunicaciones. Es entonces que conocer los perfiles de los profesionales que demanda el sector de las telecomunicaciones, permite a los centros de formación, redefinir sus objetivos de formación, con la incorporación de nuevos contenidos o adaptación de los existentes pues los procesos de formación de vanguardia, demanda sistemas de vinculación actualizados, que interactúen con el mercado laboral, para garantizar una mejor inserción laboral a sus profesionales a fin de garantizar una formación profesional acorde a las necesidades del mercado laboral.

El principal propósito de investigación es obtener información sobre las competencias que logran adquirir los estudiantes de Ingeniería en Telecomunicaciones de la Universidad Católica de Santiago de Guayaquil en contraste con el perfil que demanda la empresa pública y privada de esta área pues existe la percepción de que los nuevos profesionales egresados de la UCSG, al momento de concursar en entrevistas laborales, no cumplen a cabalidad con los perfiles profesionales que demandan las nuevas tecnologías implementadas en las empresas del sector de telecomunicaciones.

La investigación busca confirmar la hipótesis basada en la percepción que se tiene de que "Los estudiantes de ingeniería en Telecomunicaciones, no logran desarrollar las competencias que demanda la Corporación Nacional de Telecomunicaciones y las empresas privadas en esta área".

\section{Planes de estudio y perfiles profesionales}

Como lo dice el Informe Delors (1996), la formación profesional debe garantizar que el profesional pueda trabajar con lo que sabe, lo que está directamente relacionado con las competencias que ha adquirido. Zabalza (2011) y Hawes y Corvalán (2005) abordan la importancia de un plan de estudio cuyos contenidos y prácticas, respondan al perfil profesional que demanda el sector. Ambos consideran que los planes de estudio y el desarrollo de capacidades y competencias, orientados al cumplimiento de perfiles, responderán la demanda de profesionales capacitados y competentes, para asumir en condiciones óptimas, las responsabilidades propias del desarrollo de funciones y tareas de una determinada profesión. Fernández y otros, (2002 citado por Yaniz, 2004), apoyan las teorías de 
formación basada en perfiles, consideran que el perfil profesional "define la identidad profesional de las personas que, con una titulación académica, llevan a cabo una determinada labor y explica las funciones principales que dicha profesión cumple.

Como manifiesta Zabalza (2011) en los Planes de Estudio se reduce a seleccionar un elenco de disciplinas que permitan completar el número de créditos necesarios para obtener un título profesional, si quiere ser un proyecto formativo debe fundamentar bien la selección de los contenidos. Este autor resalta entre estos contenidos los culturales generales, los formativos generales, los contenidos formativos disciplinares, los contenidos especializados y el practicum.

De acuerdo a Maldonado (2011):

Un perfil profesional describe los posibles y más relevantes ámbitos de desempeño laboral, las responsabilidades que le corresponde asumir, las funciones que está en capacidad de desarrollar, los cargos que puede desempeñar, el tipo de instituciones en donde se puede desempeñar y contribuir en virtud de la formación de nivel superior que ha recibido (p.42).

Si como afirma Yaniz (2008) los programas de formación basados en competencias comprenden algunas características como enfocar la actuación a la práctica o aplicación profesional e integrar los contenidos aplicables al trabajo, el término de competencias requiere ser aclarado.

\section{Competencias para ejercer la profesión}

Como afirman Corvalán y Hawes (2006) es necesario para establecer una estrategia de rediseño curricular incluir los estándares de competencia en la enseñanza universitaria.

Guy Le Boterf (2001) menciona que la competencia es el "saber actuar en un contexto de trabajo, combinando y movilizando los recursos necesarios para el logro de un resultado excelente y que es validado en una situación de trabajo"(p.93). En esta definición se incluye entonces: a) Los conocimientos, saber, saber-hacer, saberser; b) las bases de datos, redes de expertos; c) el contexto profesional dado (organización del trabajo), y d) los resultados esperados, necesidades a satisfacer, criterios de desempeño y logros predeterminados).

El proyecto DeSeco (OCDE, 2003) definió la competencia como 
La capacidad para responder a las exigencias individuales o sociales para realizar una actividad o una tarea" desde una combinación de "habilidades prácticas y cognitivas interrelacionadas, conocimientos (incluyendo el conocimiento tácito), motivación, valores, actitudes, emociones y otros elementos sociales y de comportamiento que pueden ser movilizados conjuntamente para actuar de manera eficaz (p.8).

Como manifiesta el Espacio Europeo de Educación Superior (2009):

El concepto de competencia representa una apuesta decidida por acercar el aprendizaje a los problemas y exigencias de la vida contemporánea, al entender el conocimiento disciplinar o interdisciplinar que se trabaja en la universidad no como un fin en sí mismo, sino como un instrumentos, el instrumento privilegiado, al servicio de las competencias o cualidades humanas fundamentales, entender la realidad compleja en que vivimos; conocerse y gobernarse a sí mismo, relacionarse con los demás en contextos heterogéneos; y elaborar los propios proyectos de vida personal, social y profesional (p.14).

La competencia debe integrar "aquello que hemos de aprender, cómo hemos de aplicar y poner en práctica lo que hemos aprendido y las actitudes, emociones y valores que subyacen al proceso de enseñar y aprender" (Medina, 2009, p.13)

Para Rue (2008) las concepciones relativas a competencias se relacionan con niveles superiores de actuación laboral u ocupacional y se aprenden y desarrollar a partir de contextos.

A pesar de la diversidad de definiciones que tiene el concepto de competencia, es posible distinguir algunos rasgos característicos como: la integración de conocimientos, procedimientos y actitudes, es decir lo que el individuo ha de saber, saber hacer y saber estar para saber actuar en forma pertinente.

Pugh y Lozano-Rodríguez (2019) basados en Frade (2009) distinguen tres patrones comunes en las competencias: “a) son una capacidad cognitivo-conductual que se traduce en un desempeño, b) son una respuesta adaptativa a las demandas del entorno; c) incluyen conocimientos, capacidades, aptitudes, habilidades, destrezas" (p.149). 


\section{Materiales y métodos}

Se realizó una investigación que empleó metodología cuantitativa. La estrategia de recogida de información se basó principalmente en el uso de cuestionarios que fueron aplicados a los estudiantes, profesores y gerentes.Para el cálculo del tamaño de la población se tomó el listado de estudiantes de acuerdo a las materias cursadas en el noveno ciclo en dos periodos consecutivos, se obtuvo como tamaño de la población 57 estudiantes y se entrevistó a 50 estudiantes. Se seleccionó a los profesores que dictan las materias de especialización en la Carrera de Telecomunicaciones de la Universidad Católica Santiago de Guayaquil en el semestre A-2013. De un universo de 18 profesores que dictan materias de especialidad, es decir materias a partir del V ciclo de la carrera, se encuestaron a 10 profesores elegidos en base a las materias que los estudiantes consideraron como muy importantes para su carrera.

En el caso de los gerentes se aplicó un cuestionario a 5 gerentes del área técnica de operadoras con mayor cantidad de personal técnico tales como: CNT EP, Claro, Movistar y TV Cable.

Tabla 2.1.

Categorías de investigación

Temas Categorías

Contenidos Importancia de las materias

curriculares

Competencia Preparación

Noción del giro del negocio

Capacidades

Experiencias laborales

Manejo de Equipos

Procedimiento de Métodos Seguros

Elaboración de informes

Experiencia en Áreas donde desempeñaron sus

Pasantías actividades

Actividades desarrolladas

Materia vinculadas

Campo Lugar donde le gustaría trabajar

profesional 
Se aplicaron tres cuestionarios y se utilizaron ítems cerrados y abiertos. La encuesta para los pasantes contó con 17 preguntas. Para el caso de los profesores fueron los mismos temas con excepción del aspecto de las pasantías. La encuesta constaba de 10 preguntas. A los gerentes se realizó un cuestionario de 13 preguntas que se enfocaban en los contenidos y competencias que perciben los gerentes que tienen los ingenieros en telecomunicaciones y lo que demandan.

Para realizar el análisis de datos se utilizó una de las aplicaciones de Microsoft Office, Excel, hoja de cálculo electrónica, que permite crear bases de datos y realizar el análisis de estos.

\section{Resultados}

A continuación se presentan las dimensiones investigadas con la frecuencia medida en porcentaje.

Los contenidos curriculares son la esencia de los programas de estudios y tienen como finalidad generar los conocimientos y competencias para ejercer una profesión. En este estudio se pudo determinar que el $35 \%$ de los estudiantes encuestados considera que es muy importante la totalidad de las materias contenidas en la malla curricular de su especialidad como ingenieros en telecomunicaciones, el $34 \%$ de los estudiantes consideran imprescindible la totalidad de las materias de su malla curricular, el $26 \%$ de los estudiantes consideran las materias como importantes, el $5 \%$ poco importante, y no hubo respuestas de supresión de materias. Dentro de las materias consideradas muy importantes para los estudiantes que siguen la especialización en Telecomunicaciones están: Planta Externa, Laboratorio de Electrónica, Electrónica II, Fundamentos de Comunicación, Comunicación y Tráfico, Telemática I, Transmisión, Sistemas de Fibra óptica y Telemática II, lo cual guarda pertinencia con su especialidad formativa.

Es importante destacar que los estudiantes que consideraron como poco importantes las asignaturas, el 54\% justificó su respuesta porque consideran que no tienen la utilidad necesaria o requerida 
para su especialización, por otra parte el $31 \%$ endosan su respuesta a la forma en que el docente expuso su contenido, con poca claridad y sin motivar su importancia, además de emplear conceptos no actualizados con los avances tecnológicos, el 15\% restante considera que el contenido de estas asignaturas se encuentran en otras materias.

Respecto a la practicidad de las materias, el 56\% opina que la malla actual de la carrera de telecomunicaciones es práctica para la vida profesional, en cambio el $36 \%$ considera lo contrario y el $8 \%$ le es indiferente. Estos resultados evidencian un malestar y una falta de identidad con respecto al perfil profesional que aspiran los estudiantes adquirir.

Los profesores manifestaron que debe fortalecerse materias como: Sistemas de Televisión, Telemática, y Antenas consideran que los contenidos de las materias deben ser revisados, la principal razón que expusieron para esta acción es que son materias base para el ejercicio profesional y sus contenidos constantemente se encuentran evolucionando a nuevas tecnologías de comunicación.

Las enseñanzas de grado deben permitir que el estudiante desarrollar las actividades de carácter profesional. ¿Qué actividades pueden realizar los estudiantes? Para responder a esta interrogante, se investigó sobre la preparación para el desarrollo de actividades, la noción del giro del negocio, las capacidades, la experiencia laboral, las prácticas seguras y la elaboración de informes técnicos.

a) Preparación para el desarrollo de actividades

Una de las preguntas sobre los aspectos de la competencia, intentaba medir la percepción sobre la preparación para el desarrollo de actividades profesionales, se halló que se sienten mejor capacitados para el desarrollo de proyectos: en diseño y fiscalización en un $24 \%$, para el área de construcción de infraestructura en un 9\%, para operar las redes en un $8 \%$, para dar mantenimiento a la infraestructura existente, planificar, dirigir e implementar tecnología en un $6 \%$, mientras que para asesorar lo cual demanda un conocimiento trasversal de servicios en un 5\%. 
Desde el punto de vista de los profesores encuestados, el 23\% de ellos consideran que los estudiantes tienen la habilidad de operar proyectos de telecomunicaciones, el $15 \%$ considera que está capacitado para asesorar y fiscalizar proyectos que demandan de un grado mayor de conocimiento y experiencia. En un $8 \%$ los estudiantes están capacitados en actividades de: planificación, dirección, construcción e implementación y para labores de mantenimiento, y en un 7\% esta capacitados para diseñar proyectos de telecomunicaciones.

Para los gerentes encuestados, los profesionales contratados de la UCSG se encuentran preparados principalmente para ejercer actividades de planificación y construcción de redes, además de que consideran que pueden laborar en el área de operaciones y para efectuar tareas de fiscalización, sin embargo, deben fortalecerse en actividades técnicas, principalmente en las áreas de implementación de proyecto, de diseño de infraestructura y construcción de redes de cobre y fibra óptica.

b) Noción del giro del negocio

En la interrogante sobre la noción del giro de negocios de telecomunicaciones en el proceso de formación de los estudiantes, el $55 \%$ de los estudiantes encuestados tienen un conocimiento del mercado de telecomunicaciones, seguido de un $28 \%$ con visión empresarial, un $14 \%$ con posibilidades de iniciar sus propia empresas contratistas de implementación de infraestructura telefónica y $3 \%$ con capacidad de asesoramiento de inversiones en el sector de telecomunicaciones. En esta variable los profesores consideraron que los estudiantes se sustentan en conocimiento del mercado de telecomunicaciones en un $62 \%$ y el $38 \%$ piensa que podrían generar empresas de telecomunicaciones, lo cual evidencia una percepción muy diferente entre lo que creen los estudiantes y los profesores.

\section{c) Capacidades}

En lo que se refiere a las capacidades que debería dominar el ingeniero en telecomunicaciones, el $76 \%$ afirmó que puede desarrollar bien la actividad del cableado estructurado; un $20 \%$ se 
sintió mejor preparado para la instalación y mantenimiento de sistemas de banda ancha ADSL y el 12\% para la televisión satelital DTH. Al ser estos los nuevos servicios que ofrecen las operadoras de telefonía, se evidencia una limitación competitiva de los estudiantes, los cuales se orientan más al cableado estructurado que a los sistemas de internet y televisión, lo que no les permite cumplir el perfil profesional que demandan las operadoras de telecomunicaciones.

La principal habilidad que los profesores consideran que los estudiantes están capacitados es para el cableado estructurado (86\%), con respecto a actividades de instalación y mantenimiento de sistemas de banda ancha ADSL en un 50\% de los profesores encuestados consideran que los estudiantes pueden efectuarla, sin embargo la mayoría ( $83 \%$ ) considera que no están preparados para la televisión satelital DTH.

Los gerentes entrevistados señalaron que se encuentran preparados principalmente para ejercer actividades de planificación y construcción de redes, además de que consideran que pueden laborar en el área de operaciones y para efectuar tareas de fiscalización. Sin embargo mencionaron que no pueden realizar los diagnósticos de factibilidad (comprende localización de reservas, elección del medio de acceso cobre o fibra, diseño de acometida, cuantificación y elaboración de presupuesto) por la falta de capacitación y experiencia en el uso de equipos de medición.

\section{d) Experiencia laboral}

En función de las habilidades que han adquirido por la experiencia laboral, el $60 \%$ de los estudiantes la ha obtenido en diseño y práctica en construcción de redes ópticas, el $52 \%$ en base de datos, tablas dinámicas que le permiten tener indicadores de gestión y el $36 \%$ en equipos multiplexores. El desarrollo de competencias de acuerdo a los resultados obtenidos estaría orientados a procesos constructivos de redes y en menor escala a los procesos de planta interna que demanda el manejo de equipos multiplexores, concentradores y control estadístico de indicadores de gestión de redes. 
e) Manejo de equipo

Se halló como resultado en el manejo de los equipos que los estudiantes en un $96 \%$ tienen conocimiento de manejo de multímetros, del $20 \%$ al $28 \%$ conocen el manejo de equipos localizadores de fallas (ecómetros y OTDR), y en un $8 \%$ tienen conocimiento de manejo de equipos medidores de parámetros de aislamiento, atenuación y puesta a tierra (megger, decibelímetros y medidores de tierra). De igual forma existe un pequeño grupo que no conocen los equipos en cuanto a su manejo al 100\% siendo su razón principal la falta de equipamiento en los laboratorios en donde realizan las prácticas. Esta realidad evidencia una falta de competencia y del perfil profesional que requieren cumplir para aspirar una plaza laboral en aquellas empresas donde la calidad de los servicios que prestan están asociadas al servicio continuo y en la velocidad de respuesta que brindan a sus usuarios en la atención de interrupciones de servicios, lo cual requiere que personal técnico que la conforma tenga conocimiento y experticia en el manejo de equipos localizadores de fallas y verificación de parámetros ópticos y eléctricos.

Todos los profesores coinciden que si pueden manejar un multímetro. La mitad considera que puede manejar los meger (medidores de aislamiento), pero los docentes piensan que otros equipos como el ecómetro, OTDR, decibelímetros y medidores de tierra no están capacitados pues sus respuestas afirmativas fueron menores del 33\%. Lo cual evidencia que los estudiantes no se encuentran preparados para el uso de equipos detectores de fallas.

\section{f) Prácticas seguras}

En lo concerniente a los procedimientos y métodos seguros, el $80 \%$ de los estudiantes no conocen acerca de la protección personal en cuanto al uso de un equipo adecuado a sus tareas de trabajo. Lo cual evidencia que los estudiantes no se encuentran preparados para realizar actividades seguras de trabajo en el campo profesional, los equipos de protección personal no evitan accidentes, pero minimizan que los factores de riesgo que pueden ocasionar lesiones o enfermedades a los trabajadores, en la actualidad el uso de los equipos de protección personal es obligatorio y su incumplimiento 
puede desproteger al trabajador de reclamaciones al seguro social, lo cual demanda de la revisión de la malla que incluya una asignatura orientadas al manejo de equipos de seguridad y a evitar situaciones inseguras de trabajos y factores de riesgo laboral.

g) Elaboración de informes

Cabe resaltar que el $76 \%$ de los estudiantes encuestados considera estar preparado para realizar informes técnicos de gestión en su desempeño del área de trabajo, frente al $24 \%$ que considera lo contrario

\subsection{Pasantías}

El practicum tiene como objetivo integrar al estudiante en un contexto de aprendizaje situado en campos reales relacionados con la práctica del rol profesional a desempeñar pues el alumno requiere adquirir conocimientos, información, habilidades y competencias para el ejercicio profesional (Villa y Poblete, 2004). En esta investigación se abordó este tema para averiguar las competencias que habían adquirido. Se indagó sobre las áreas en donde se desarrollaron, las actividades realizadas y las materias que aportaron en esta práctica.

a) Áreas donde desarrollaron las pasantías

Los estudiantes encuestados destacaron que el $44 \%$ de las actividades que conforman el sector de planta externa conocieron al momento de realizar sus pasantías, el $26 \%$ de las actividades relacionadas a planta interna o centrales telefónicas, frente al $30 \%$ del sector de transmisiones. Si las pasantías es lo más cercano al ejercicio profesional, lo cual le permite al estudiante familiarizarse con los escenarios de trabajo y con las condiciones reales que se imponen como trabajadores, el hecho de que los estudiantes consideren que sus pasantías mayoritariamente fueron orientadas a actividades relacionadas con planta externa (Telefonía Fija), limitan su experiencia práctica con respecto a las otras áreas de servicios que ofrecen las operadoras de telecomunicaciones tales como internet y televisión, lo cual afecta significativamente a los recién 
graduados al momento de participar en un concurso de selección de personal.

b) Actividades desarrollas en las pasantías

Se encontró que en la pasantía, los estudiantes desarrollaron en un porcentaje igual del $28 \%$ tanto las actividades de cableado como el diseño de redes, seguido de manejo de fibra óptica en un $24 \%$; en el despliegue de tareas relacionadas con nodos o mini centrales participaron el $16 \%$ de los estudiantes y solo el 4\% dedicó las actividades de sus pasantías al manejo de antenas parabólicas DTH. Estos hallazgos evidencian que las funciones realizadas por los pasantes, está orientadas a procesos de diseño y construcción de planta externa y alimentación de centrales por medios ópticos, pero no se consideran los procesos de mantenimiento y detección de fallas, así como tampoco trabajan en áreas de despliegue de sistemas de trasmisión de datos y televisión DTH, donde se realizan actividades de factibilidad, configuraciones de equipos, mediciones eléctricas y empleo de equipos localizadores de fallas.

c) Materias que aportan a las pasantías

Se indagó también sobre la asignatura que los estudiantes estimaron que aportó significativamente a desarrollar sus pasantías, se encontró que fue la materia de Planta Externa con un 51\%, seguida de Transmisión en un 11\%. Otras materias como Conmutación y Tráfico, Fundamentos de Ingeniería, Líneas de Transmisión les asignaron por igual un porcentaje del 5\%. Otras disciplinas mencionadas con un porcentaje del 3\% para cada una fueron: Laboratorio Electrónico, Digitales, Propagación, Fibra óptica, Sistemas de Fibra Óptica, Análisis y Evaluación de Proyectos, Administración de Riesgo y Digitales II. De conformidad a las respuestas obtenidas se observa que las materias que más soportaron para realizar las prácticas, están orientadas a servicios de telefonía fija, y en menor grado a las otras actividades de prestación de servicios de internet y televisión. 


\subsection{Campo profesional}

Interesaba conocer las expectativas laborales, es decir las áreas en las cuales les gustaría ejercer la profesión, los resultados revelaron una preferencia por trabajar en las operadoras telefónicas privadas Claro, Movistar, como lo señaló el 31\% de los encuestados, seguido con una diferencia mínima en un porcentaje de $30 \%$ en la empresa pública CNT EP, un 13\% le gustaría formar parte de los servicios de televisión pagada como TV CABLE, UNIVISA, DIRECTV, un $10 \%$ prefiere trabajar en servicios de internet o tener su propia empresa contratista, y el 5\% en el área de radiodifusión. Como motivaciones para desear laborar en estas instituciones manifestaron en un $38 \%$ por el conocimiento que pueden obtener de otras personas que ya laboran ahí con más tiempo que ellos; el 20\% para adquirir experiencia personal, el $26 \%$ por un logro personal que se transforma en una satisfacción, el $10 \%$ por desarrollo profesional, el $4 \%$ por su carrera y el $2 \%$ por que les tocó trabajar ahí.

Si revisamos las expectativas de los profesores en el área que los estudiantes podrían ejercer su profesión, en un 36\% consideraron que estarían preparados para trabajar en operadoras de telefónicas privadas como Movistar y Claro, un $29 \%$ en la empresa pública CNT EP, el 21\% en operadoras de servicios de televisión, el 7\% en radiodifusión y empresas que brindan servicios móviles y el $7 \%$ en empresas propias, no se obtuvieron opiniones con respecto a empresas de servicios de internet.

\section{Discusión}

Esta realidad evidencia una falta de competencia y del perfil profesional que requieren cumplir para aspirar una plaza laboral en aquellas empresas donde la calidad de los servicios que prestan están asociadas al servicio continuo y en la velocidad de respuesta que brindan a sus usuarios en la atención de interrupciones de servicios, lo cual requiere que personal técnico que la conforma tenga conocimiento y experticia en el manejo de equipos localizadores de fallas y verificación de parámetros ópticos y eléctricos.

Los centros de educación superior deben de implementar procesos permanente de actualización de conocimientos, tal como se realizan 
en la mayoría de los países europeos generadores de tecnología, donde los títulos otorgados tienen vigencia cinco años, pasado los cuales los profesionales deben aprobar pruebas de actualización de conocimientos, para que sus títulos sean validados por otro periodo de cinco años.

La investigación no considero en el estudio, la inserción laboral de los profesionales

graduados en los cinco últimos años para establecer tendencias de aceptación profesional, además no se realizaron comparaciones con otros estudios similares que soporten los resultados obtenidos.

\section{Conclusiones}

Se concluye que los contenidos de las materias que conforman el currículum actual de la carrera de Ingeniería en Telecomunicaciones de la Universidad Católica Santiago de Guayaquil, debe ser revisado en temas específicos y de últimas tecnologías, existen vacíos que por falta de actualización permanente de los contenidos de las materias de especialización, no son abordados temas tales como: implementación de servicios de banda ancha, instalación y mantenimiento de equipos multiplexores, diseño, implementación de redes ópticas pasivas, entre otros temas.

Las pasantías, están deben ser conceptualizadas bajo un paradigma integral de formación progresiva ya que es un componente sustantivo del proceso de formación universitaria, donde los contenidos de las materias que conforman el currículum actual de la carrera de Ingeniería en Telecomunicaciones de la Universidad Católica Santiago de Guayaquil debe estar orientado a dar el soporte conceptual de las prácticas en los diferentes servicios de comunicación, los estudiantes se encuentran con limitaciones o falta de conocimientos en lo que respecta a calibración de equipos y trabajo en grupos, también se demanda conocimientos de utilización de EPP (Equipos de protección personal) para evitar realizar actividades que generen condiciones inseguras de trabajo.

Las competencias que demandan las empresas públicas y privadas de telecomunicaciones se pudo establecer las siguientes: El Ingeniero en Telecomunicaciones debe tener conocimientos en 
planta externa, transmisión, gestión de redes alámbricas e inalámbricas, equipamiento Dslam para servicios de internet, sistemas de televisión satelital, análisis y evaluación de proyectos, microcontroladores, sistemas de redes ópticas pasivas y activas, domótica, entre otros. Con relación a las actividades deben poder operar centrales telefónicas, nodos de acceso, y servidores de gestión, tareas de mantenimiento preventivo y correctivo, empleo de equipos localizadores de falla, fiscalización y administración de proyectos. Adicional a estas competencias y actividades las empresas exigen habilidades interpersonales como la proactividad, liderazgo y trabajo en equipo.

\section{Referencias}

Corvalán, O y Hawes, G. (2006). Aplicación del enfoque de competencias en la construcción curricular de la Universidad de Talca. Revista Iberoamericana de Educación, 40 (1), 1-17.

Delors, J. (1996). Informe Delors: La educación encierra un tesoro. Madrid, España: Unesco Santillana.

Espacio Europeo de Educación Superior (2009). Los títulos universitarios y las competencias fundamentales: Los tres ciclos. Madrid, España: Junta de Andalucía-Akal.

Le Boterf, G. (2001). Ingeniería de las competencias. Barcelona, España: Ediciones Gestión 2000.

Hawes G y Corvalán, O. (2005). Construcción de un perfil profesional. Universidad de Talca. Instituto de Investigación y Desarrollo Educacional. Proyecto Mecesup Talo101: Universidad de Talca. Recuperado de http://vrdp.utalca.cl/docs/pdf/Construccion_Perfil_Profesional.pdf

Maldonado, M. (2011). Currículo con enfoque de competencia. Bogotá, Colombia: Ecoediciones.com.

Medina, A. (2009). Fundamentación de las competencias discentes y docentes. En A. Medina (Ed.), Formación y desarrollo de la competencias básicas (pp. 11-44). Madrid, España: Universitas. 
Organización para la Cooperación y el Desarrollo Económico, OCDE (2003). La Definición y selección de competencia clave. Resumen Ejecutivo. Paris, Francia: OCDE.

Pugh, G. y Lozano-Rodríguez, A. (2019). El desarrollo de competencias genéricas en la educación técnica de nivel superior: un estudio de caso. Calidad en la educación, (50), 143-179. doi:https://doi.org/10.31619/caledu.n50.725

Rué, J. (2008). Formar en competencias en la universidad: entre la relevancia y la banalidad. REDU. Revista de Docencia Universitaria, 6(1), 119.

Yániz, C. (2004). Convergencia europea de las titulaciones universitarias. El proceso de adaptación, fases y tareas. Revista de la Red Estatal de docencia universitaria. 4(1), 3-14.

Yániz, C. (2008). Las competencias en el currículo universitario: implicaciones para diseñar el aprendizaje y para la formación del profesorado. REDU Revista de Docencia Universitaria, 6(1), 1-14.

Villa, A y Poblete, M. (2004). Practicum y Evaluación de Competencias. Profesorado, revista de Curriculum y Formación del Profesorado, 8(2), 1-19.

Zabalza, M. (2011). Competencias docentes del Profesor Universitario. Calidad y desarrollo profesional. Madrid, España: Narcea. 